\title{
Application of ADM Using Laplace Transform to Approximate Solutions of Nonlinear Deformation for Cantilever Beam
}

\author{
Ratchata Theinchai, Siriwan Chankan, and Weera Yukunthorn \\ Faculty of Science and Technology, Kanchanaburi Rajabhat University, Kanchanaburi 71000, Thailand \\ Correspondence should be addressed to Weera Yukunthorn; weera.mec@gmail.com
}

Received 27 May 2016; Accepted 8 August 2016

Academic Editor: Birendra Nath Mandal

Copyright (C) 2016 Ratchata Theinchai et al. This is an open access article distributed under the Creative Commons Attribution License, which permits unrestricted use, distribution, and reproduction in any medium, provided the original work is properly cited.

We investigate semianalytical solutions of Euler-Bernoulli beam equation by using Laplace transform and Adomian decomposition method (LADM). The deformation of a uniform flexible cantilever beam is formulated to initial value problems. We separate the problems into 2 cases: integer order for small deformation and fractional order for large deformation. The numerical results show the approximated solutions of deflection curve, moment diagram, and shear diagram of the presented method.

\section{Introduction}

The Euler-Bernoulli beam theory states that the action load produces the bending moment $M(x) \in C([0, L])$ which is proportional to deflection characteristics of the beam. The equation of this law can be written as follows:

$$
\frac{y^{\prime \prime}}{\left[1+\left(y^{\prime}\right)^{2}\right]^{3 / 2}}=-\frac{M(x)}{E I},
$$

where $y \in C^{2}([0, L])$ is deflection curve of a uniform beam, the modulus of elasticity $E$, and the moment of inertia $I$. We note that $E$ and $I$ are both constant and the product of $E$ and $I$ is called beam stiffness. In a case of small deformation, we assume that $y^{\prime}(x)$ is infinitesimal. Equation (1) is reduced to the well-known fourth-order linear differential equation:

$$
\text { EI } \frac{d^{4} y}{d x^{4}}=\frac{d^{2} M}{d x^{2}} .
$$

In this study we consider the uniform flexible of cantilever beam; see Figure 1 . The parameters $L$ and $\Delta$ are undeformed length and horizontal displacement, respectively. The deformed length of beam is verified by the integral $\int_{0}^{l}\left[1+\left(y^{\prime}\right)^{2}\right]^{1 / 2} d x$, where $l=L-\Delta$. It was shown in [1] that the slope of deflection curve represents the following equation $y^{\prime}(x)=G(x) /\left[1-G^{2}(x)\right]^{1 / 2}$, where $G(x)=$ $\left(\int_{x}^{l} M(s) d s\right) /(E I)$ for the known function $M$. The deflection curve in Figure 1 corresponds to initial value problem of the geometric problem:

$$
\begin{aligned}
\frac{y^{\prime \prime}(x)}{\left[1+\left(y^{\prime}(x)\right)^{2}\right]^{3 / 2}} & =-\frac{M(x)}{E I}, \quad x \in[0, l] \\
y(0) & =0, \\
y^{\prime}(0) & =\frac{G(0)}{\left[1-G^{2}(0)\right]^{1 / 2}},
\end{aligned}
$$

where $M(x)=P x$. If the slope is very small, the linear EulerBernoulli beam theory [2] governs the problem

$$
\begin{aligned}
E I \frac{d^{4} y}{d x^{4}} & =0, \quad x \in[0, L], \\
E I y^{\prime \prime}(0) & =-P L, \\
E I y^{(3)}(0) & =-P, \\
y(L) & =\frac{P L^{3}}{3 E I}, \\
y^{\prime}(L) & =0 .
\end{aligned}
$$




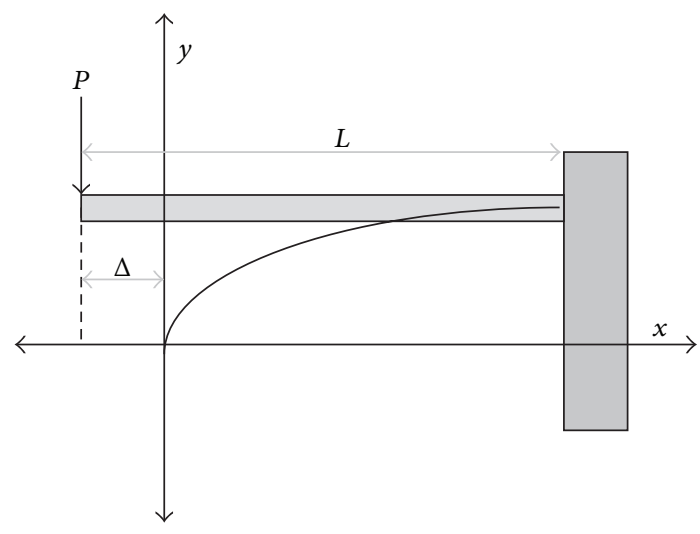

FIgURE 1: Cantilever beam with a concentrated load at the free end.

The solution of the problem (4) is

$$
y(x)=\frac{P x^{3}}{6 E I}-\frac{P L^{2} x}{2 E I} .
$$

Our method proposes to reform problem (3) in a sense of fractional calculus without linearization. Because fractional calculus is the great idea to describe behavior in nature, for example, force response of viscoelastic material $[3,4]$, fluid flow [5], and fitting experimental data [6]. For convenience, we set $k=-P / E I$ and $c=G(0) /\left[1-G^{2}(0)\right]^{1 / 2}$. We develop (1) to deal with fractional order $\alpha \in(1,2]$; system (3) becomes

$$
\begin{aligned}
D_{0}^{\alpha} y(x) & =k x\left[1+\left(y^{\prime}(x)\right)^{2}\right]^{3 / 2}, \quad x \in[0, l], \\
y(0) & =0 \\
y^{\prime}(0) & =c .
\end{aligned}
$$

We use Adomian polynomial to approximate a nonlinear term and derive a semianalytical solution by use of Laplace transform for the initial value problem (6).

\section{Preliminaries}

In this section we introduce some definitions and theory of fractional calculus and Laplace transform which are used in our method. See [7] for more details.

Definition 1. The Riemann-Liouville fractional integral operator of order $\alpha>0$ is defined as

$$
I_{0}^{\alpha} y(x)=\frac{1}{\Gamma(\alpha)} \int_{0}^{t}(t-\tau)^{\alpha-1} f(\tau) d \tau .
$$

Definition 2. The Caputo fractional derivative of order $\alpha>0$ is defined as

$$
\begin{array}{r}
D_{0}^{\alpha} f(t)=\frac{1}{\Gamma(n-\alpha)} \int_{0}^{t}(t-\tau)^{n-\alpha-1} f^{(n)}(\tau) d \tau \\
\quad n=\lceil\alpha\rceil .
\end{array}
$$

Theorem 3. If $p>0$ and $q>0$, then $\left(D_{0}^{p} D_{0}^{q} f\right)(x)=$ $D^{p+q} f(x)$.

Theorem 4. The Laplace transform of the Caputo fractional derivative is given by

$$
L\left\{D_{0}^{\alpha} y(x)\right\}=s^{\alpha} L\{y(x)\}-\sum_{k=0}^{n} s^{\alpha-k-1} y^{(k)}(0),
$$

where $n=\lfloor\alpha\rfloor$ and $k$ is a nonnegative integer. The inverse Laplace transform of power function is given by

$$
L^{-1}\left\{\frac{\Gamma(p+1)}{s^{p+1}}\right\}=t^{p}, \quad p>-1 .
$$

\section{Semianalytical Solution via LADM}

To formulate the general solution of problem (6), we replace $y^{\prime}$ with $u$ in the equation of problem (6) and applied Theorem 3. We obtain

$$
D_{0}^{\alpha-1} u=k x\left[1+u^{2}\right]^{3 / 2}
$$

Taking Laplace transform of (11) gives

$$
L\left\{D_{0}^{\alpha-1} u\right\}=k L\left\{x\left[1+u^{2}\right]^{3 / 2}\right\} .
$$

Using Theorem 4 and replacing $y^{\prime}(0)$ by $c$, we can rewrite as

$$
L\{u\}=\frac{c}{s}+k s^{1-\alpha} L\left\{x\left[1+u^{2}\right]^{3 / 2}\right\} .
$$

We take inverse Laplace transform of (13), and the following operator equation is obtained:

$$
u=c+k L^{-1}\left\{s^{1-\alpha} L\left\{x\left[1+u^{2}\right]^{3 / 2}\right\}\right\} .
$$

We apply Adomian polynomial $[8,9]$ to approximate a nonlinear term of (14), by setting

$$
\begin{gathered}
N u=\left[1+u^{2}\right]^{3 / 2}=\sum_{n=0}^{\infty} A_{n}(u) ; \\
A_{n}(u)=\frac{1}{n !}\left(\sum_{i=0}^{\infty} c(v, n) \frac{d^{v} N u}{d u^{v}}\right) .
\end{gathered}
$$

Equation (14) becomes

$$
u=c+k L^{-1}\left\{s^{1-\alpha} L\left\{x \sum_{n=0}^{\infty} A_{n}(u)\right\}\right\} .
$$

Assume that the solution of (11) can be written as $u=\sum_{n=1}^{\infty} u_{n}$. From the combination of Laplace transform and Adomian decomposition method, we obtain the formulation of LADM for fractional order of cantilever beam deflection equation:

$$
\sum_{n=0}^{\infty} u_{n}=c+k L^{-1}\left\{s^{1-\alpha} L\left\{x \sum_{n=0}^{\infty} A_{n}(u)\right\}\right\} .
$$


In this work, we apply the first four terms of LADM which are provided from following recurrence:

$$
\begin{aligned}
u_{0} & =c, \\
u_{i+1} & =k L^{-1}\left\{s^{1-\alpha} L\left\{x A_{i}\right\}\right\},
\end{aligned}
$$

for $i=0,1,2$,

where

$$
\begin{aligned}
& A_{0}=N u_{0}, \\
& A_{1}=u_{1} \frac{d\left(N u_{0}\right)}{d u_{0}}, \\
& A_{2}=u_{2} \frac{d\left(N u_{0}\right)}{d u_{0}}+\frac{u_{1}^{2}}{2 !} \frac{d^{2}\left(N u_{0}\right)}{d u_{0}^{2}} .
\end{aligned}
$$

The components of adomian polynomial are given by

$$
\begin{aligned}
A_{0}= & \left(1+u_{0}^{2}\right)^{3 / 2} \\
A_{1}= & 3 u_{0} u_{1}\left(1+u_{0}^{2}\right)^{1 / 2}, \\
A_{2}= & 3 u_{0} u_{2}\left(1+u_{0}^{2}\right)^{1 / 2}+\frac{3}{2} u_{1}^{2}\left(1+u_{0}^{2}\right)^{1 / 2} \\
& +\frac{3}{2}\left(u_{0} u_{1}\right)^{2}\left(1+u_{0}^{2}\right)^{-1 / 2} .
\end{aligned}
$$

The iteration results of recurrence (18) are shown as follows:

$$
\begin{aligned}
u_{0} & =c \\
A_{0} & =\left(1+c^{2}\right)^{3 / 2}, \\
u_{1} & =-k L^{-1}\left\{s^{1-\alpha} L\left\{x A_{0}\right\}\right\}=-\frac{k\left(1+c^{2}\right)^{3 / 2}}{\Gamma(1+\alpha)} x^{\alpha}, \\
A_{1} & =-\frac{3 c k\left(1+c^{2}\right)^{2}}{\Gamma(2+\alpha-1)} x^{\alpha}, \\
u_{2} & =-k L^{-1}\left\{s^{1-\alpha} L\left\{x A_{1}\right\}\right\}=\frac{3 c k^{2}\left(1+c^{2}\right)^{2} \Gamma(2+\alpha)}{\Gamma(1+\alpha) \Gamma(1+2 \alpha)} \\
& \cdot x^{2 \alpha}, \\
A_{2} & =\left(\frac{9 c^{2} \Gamma(2+\alpha)}{\Gamma(1+2 \alpha)}+\frac{3\left(1+2 c^{2}\right)}{2 \Gamma(1+\alpha)}\right) \frac{k^{2}\left(1+c^{2}\right)^{5 / 2}}{\Gamma(1+\alpha)} \\
& \cdot x^{2 \alpha},
\end{aligned}
$$

$$
\begin{aligned}
u_{3} & =-k L^{-1}\left\{s^{1-\alpha} L\left\{x A_{2}\right\}\right\} \\
& =-\left(\frac{9 c^{2} \Gamma(2+\alpha)}{\Gamma(1+2 \alpha)}+\frac{3\left(1+2 c^{2}\right)}{2 \Gamma(1+\alpha)}\right) \\
& \cdot \frac{\Gamma(2+2 \alpha) k^{3}\left(1+c^{2}\right)^{5 / 2}}{\Gamma(1+\alpha) \Gamma(1+3 \alpha)} x^{3 \alpha}
\end{aligned}
$$

The first four terms' approximate solution is $u=u_{0}+u_{1}+u_{2}+$ $u_{3}$ :

$$
\begin{aligned}
u(x)= & c-\frac{k\left(1+c^{2}\right)^{3 / 2}}{\Gamma(1+\alpha)} x^{\alpha} \\
+ & \frac{3 c k^{2}\left(1+c^{2}\right)^{2} \Gamma(2+\alpha)}{\Gamma(1+\alpha) \Gamma(1+2 \alpha)} x^{2 \alpha} \\
- & \left(\frac{9 c^{2} \Gamma(2+\alpha)}{\Gamma(1+2 \alpha)}+\frac{3\left(1+2 c^{2}\right)}{2 \Gamma(1+\alpha)}\right) \\
& \cdot \frac{\Gamma(2+2 \alpha) k^{3}\left(1+c^{2}\right)^{5 / 2}}{\Gamma(1+\alpha) \Gamma(1+3 \alpha)} x^{3 \alpha} .
\end{aligned}
$$

Since $u=y^{\prime}$ and $y(0)=0$, thus $y(x)=\int_{0}^{x} u(s) d s$. This yields

$$
\begin{aligned}
y(x)= & c x-\frac{k\left(1+c^{2}\right)^{3 / 2}}{\Gamma(2+\alpha)} x^{1+\alpha} \\
& +\frac{3 c k^{2}\left(1+c^{2}\right)^{2} \Gamma(2+\alpha)}{\Gamma(1+\alpha) \Gamma(2+2 \alpha)} x^{1+2 \alpha} \\
- & \left(\frac{9 c^{2} \Gamma(2+\alpha)}{\Gamma(1+2 \alpha)}+\frac{3\left(1+2 c^{2}\right)}{2 \Gamma(1+\alpha)}\right) \\
& \cdot \frac{\Gamma(2+2 \alpha) k^{3}\left(1+c^{2}\right)^{5 / 2}}{\Gamma(1+\alpha) \Gamma(2+3 \alpha)} x^{1+3 \alpha} .
\end{aligned}
$$

3.1. Integer Order for Small Deformation. We simulate some deflection curve of the results in (24) and (5) which are solution of LADM and classical method, respectively. The parameters are $L=100$ in., $\Delta=0$ in., $E I=1.8 \times 10^{5}$ kip in. ${ }^{2}$, $\alpha=2$, and $P=1$ kip. Figure 2 shows the deflection of cantilever beam, obtained by using classical method and LADM. LADM has accurate slope around the free end of the beam as well as classical method. Moreover LADM shows that the deflection curve remains nearly straight line in the case of small deformation.

The effects of various loads influencing maximum deflection at the free end and length of the deformed beam 


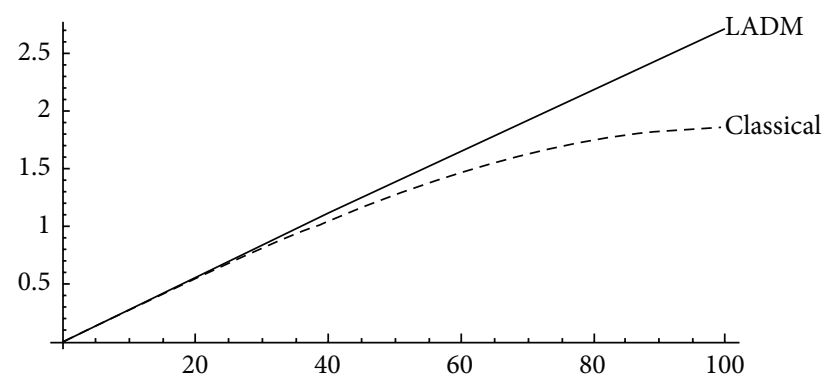

Figure 2: Small deflection curve.

TABLE 1: Numerical results under various load testing.

\begin{tabular}{lcccc}
\hline $\begin{array}{l}\text { Load } \\
\text { (kip) }\end{array}$ & $\begin{array}{c}y_{\max } \\
\text { (in.) }\end{array}$ & $\begin{array}{c}\text { Curve length } \\
\text { (in.) }\end{array}$ & $\begin{array}{c}y_{\max } \\
\text { (in.) }\end{array}$ & $\begin{array}{c}\text { Curve length } \\
\text { (in.) }\end{array}$ \\
\hline 0.1 & 0.1852 & 100.0002 & 0.2770 & 100.0004 \\
0.2 & 0.3704 & 100.0008 & 0.5525 & 100.0015 \\
0.3 & 0.5556 & 100.0019 & 0.8264 & 100.0034 \\
0.4 & 0.7407 & 100.0033 & 1.0989 & 100.0060 \\
0.5 & 0.9259 & 100.0051 & 1.3698 & 100.0094 \\
1 & 1.8519 & 100.0206 & 2.7020 & 100.0365 \\
5 & 9.2593 & 100.5125 & 12.0619 & 100.7396 \\
10 & 18.519 & 102.0283 & 20.0049 & 102.2721 \\
50 & 92.5926 & 140.3049 & - & - \\
100 & 185.1852 & 216.3719 & - & - \\
\hline
\end{tabular}

are presented in Table 1. We observe that the calculation of nonlinear integer order deformation via LADM fails at $P=50$ kips and $P=100$ kips. Since $G(0)>1$ thus integral of bending moment is greater than stiffness of the beam. This yields large deformation, large rotation, and large strain, namely, some concept of large deformation. Not only does LADM fails, but also classical method does, because it contradicts the assumption that the lengths of deformed and undeformed beam are identical. This is the motivation for reform in fractional order model.

3.2. Fractional Order for Large Deformation. There are many methods for describing a large deformation of a beam, for example, the Laplace-Padé coupling with NDHPM and HPM [10], VIM [11], and pseudolinear system [1]. In this section, we introduce the process of investigating semianalytical solution via LADM and some numerical results of pseudolinear system in [1].

To illustrate the numerical results we suppose that horizontal displacement $\Delta=40$. The beam parameters are $L=1000 \mathrm{in}$. and $E I=1.8 \times 10^{5} \mathrm{kip} \mathrm{in.}{ }^{2}$. The fact from our assumption is

$$
y^{\prime}(840)=0
$$

In this case we fixed the concentrate load $P=0.4$ kip. We get the deformed length $l=840$ and initial condition
TABLE 2: Values of slope at $840 \mathrm{in}$. and maximum vertical displacement for various fractional orders.

\begin{tabular}{lcc}
\hline Fractional order & Slope at 840 in. & Deformed length (in.) \\
\hline 1.56 & -0.185702 & 768.244392 \\
1.55 & -0.102380 & 798.176219 \\
1.54 & -0.023820 & 829.711010 \\
1.53 & 0.050256 & 862.954991 \\
1.52 & 0.120111 & 898.022706 \\
\hline
\end{tabular}

TABLE 3: Numerical results of slope deflection.

\begin{tabular}{lcc}
\hline$x$ (in.) & Pseudolinear (in.) & LADM (in.) \\
\hline 0 & 1.1049 & 1.2630 \\
100.0 & 1.0698 & 1.2132 \\
200.0 & 0.9720 & 1.1186 \\
300.0 & 0.8360 & 0.9943 \\
400.0 & 0.6822 & 0.8460 \\
500.0 & 0.5232 & 0.6773 \\
600.0 & 0.3632 & 0.4903 \\
700.0 & 0.2009 & 0.2862 \\
800.0 & 0.0304 & 0.0679 \\
816.9 & 0 & 0.0294 \\
\hline
\end{tabular}

$c=y^{\prime}(0)=1.2626$. Fractional order is determined by simulating the results of slope at 840 and deformed length. Table 2 shows that order $\alpha=1.54$ is given, $y^{\prime}(840)=-0.0238$ and $y^{\prime}(829.711)=0$, which is the most accurate for the fact in (25). We design order $\alpha=1.54$ for this problem. Using the first four terms' approximation, the deflection is shown in Figure 3(a). The slope deflection of psudolinear system and LADM are presented in Table 3. Moment diagram $\left(M_{e}\right)$ can be computed from the deflection curve as follows: $M_{e}(x)=E I y^{\prime \prime}(x) /\left[1+\left(y^{\prime}(x)\right)^{2}\right]^{3 / 2}$ and $V(x)=M_{e}^{\prime}(x)$ for shear diagram. The results of both moment and shear diagram are shown in Figures 3(b) and 3(c), respectively.

\section{Conclusion}

In this study, we use Euler-Bernoulli beam equation for describing the uniform flexible cantilever beam with a concentrated load. The initial conditions are given by calculating slope of the beam. We use LADM to determine the semianalytical solution. LADM with integer order system can approximate solution without cancellation a nonlinear term in the case of small deformation. For the large deformation, we reform the problem to fractional order system and estimate fractional order $\alpha$ which conserves the fact from the assumption. Finally, we show that LADM gives the solution as a polynomial expression which is the advantage for analyzing moment and shear diagrams. LADM may be a powerful and successive method for solving nonlinear science and engineering problem. 


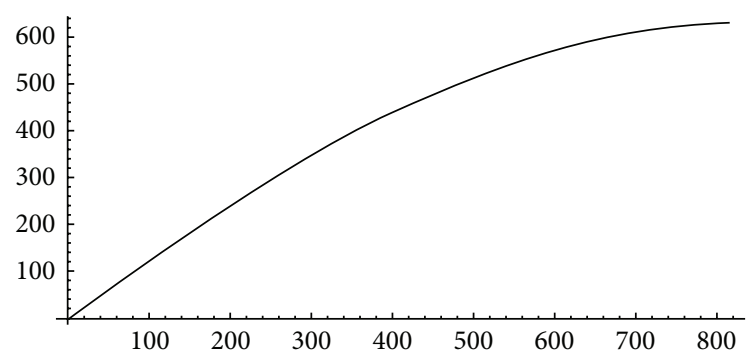

(a) Large deflection curve

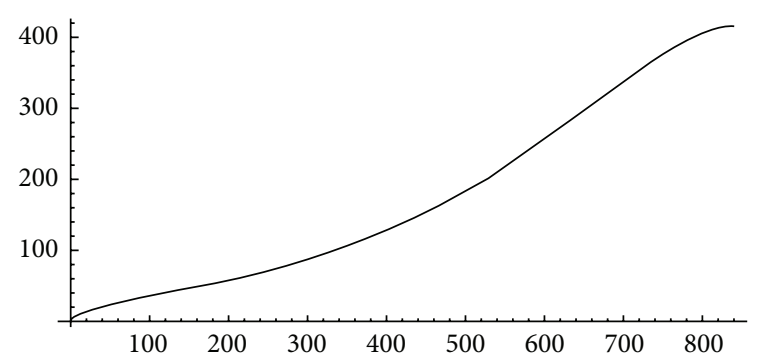

(b) Moment diagram

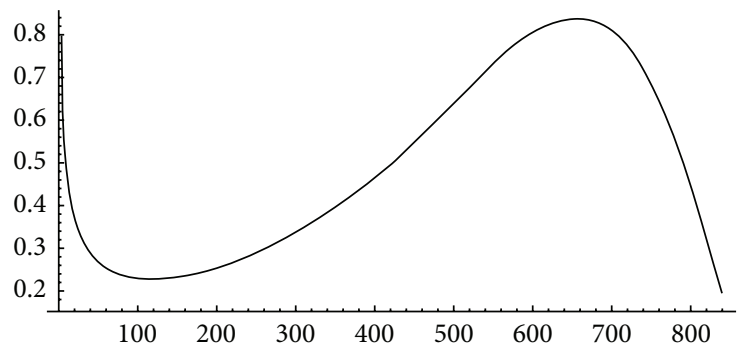

(c) Shear diagram

FIGURE 3: Numerical results of deformation via LADM (4 terms).

\section{Competing Interests}

The authors declare that there is no conflict of interests regarding the publication of this paper.

\section{References}

[1] D. G. Fertis, Nonlinear Structural Engineering: With Unique Theories and Methods to Solve Effectively Complex Nonlinear Problems, Springer, New York, NY, USA, 2006.

[2] A. F. Bower, Applied Mechanics of Solids, CRC Press, Boca Raton, Fla, USA, 2010.

[3] M. Fukunaga, N. Shimizu, and H. Nasuno, "A nonlinear fractional derivative model of impulse motion for viscoelastic materials," Physica Scripta, vol. 136, Article ID 014010, 6 pages, 2009.

[4] F. Mainardi, Fractional Calculus and Waves in Linear Viscoelasticity, Imperial College Press, London, UK, 2010.

[5] P. Lino, G. Maione, and F. Saponaro, "Fractional-order modeling of high-pressure fluid-dynamic flows: an automotive application," IFAC-PapersOnLine, vol. 48, no. 1, pp. 382-387, 2015.

[6] M. Di Paola, A. Pirrotta, and A. Valenza, "Visco-elastic behavior through fractional calculus: an easier method for best fitting experimental results," Mechanics of Materials, vol. 43, no. 12, pp. 799-806, 2011.

[7] A. A. Kilbas, H. M. Srivastava, and J. J. Truillo, Theory and Applications of Fractional Differential Equations, Elsevier, Amsterdam, The Netherlands, 2006.

[8] G. Adomian, "A review of the decomposition method in applied mathematics," Journal of Mathematical Analysis and Applications, vol. 135, no. 2, pp. 501-544, 1988.

[9] A. M. WazWaz and M. S. Mehanna, "The combined LaplaceAdomian method for handing singular integral equation of heat transfer," International Journal of Nonlinear Sciences, vol. 10, no. 2, pp. 248-252, 2010.
[10] H. Vázquez-Leal, Y. Khan, A. L. Herrera-May et al., "Approximations for large deflection of a cantilever beam under a terminal follower force and nonlinear pendulum," Mathematical Problems in Engineering, vol. 2013, Article ID 148537, 12 pages, 2013.

[11] H. Ghaffarzadeh and A. Nikkar, "Explicit solution to the large deformation of a cantilever beam under point load at the free tip using the variational iteration method-II," Journal of Mechanical Science and Technology, vol. 27, no. 11, pp. 3433-3438, 2013. 


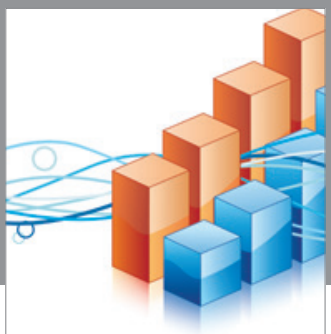

Advances in

Operations Research

vatem alat4

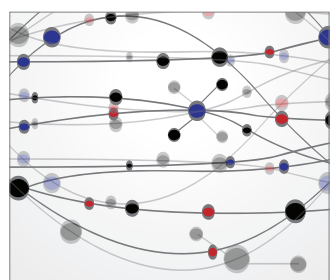

\section{The Scientific} World Journal
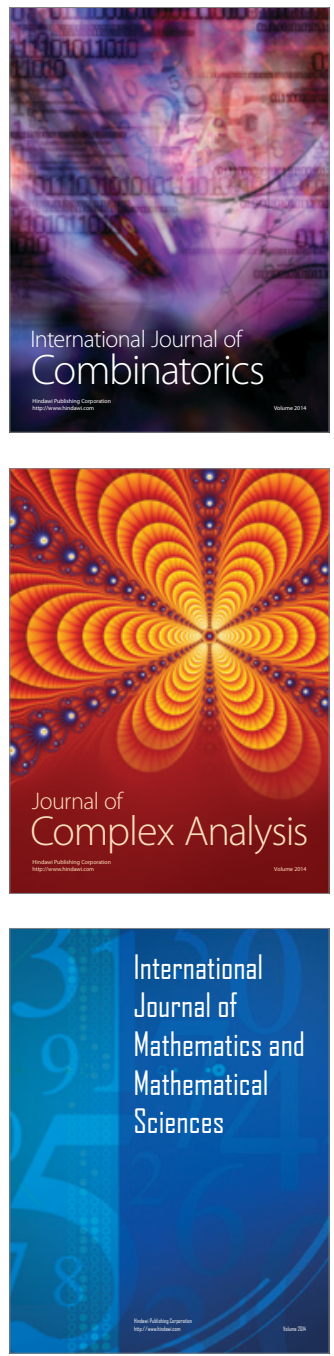
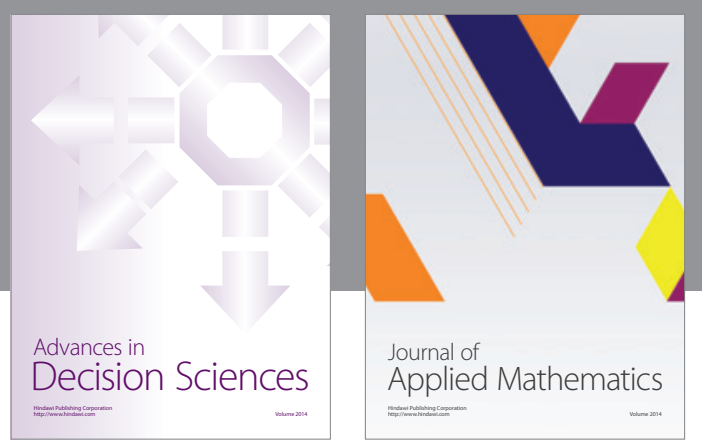

Algebra

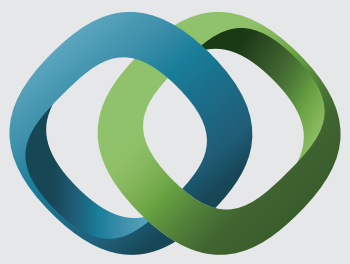

\section{Hindawi}

Submit your manuscripts at

http://www.hindawi.com
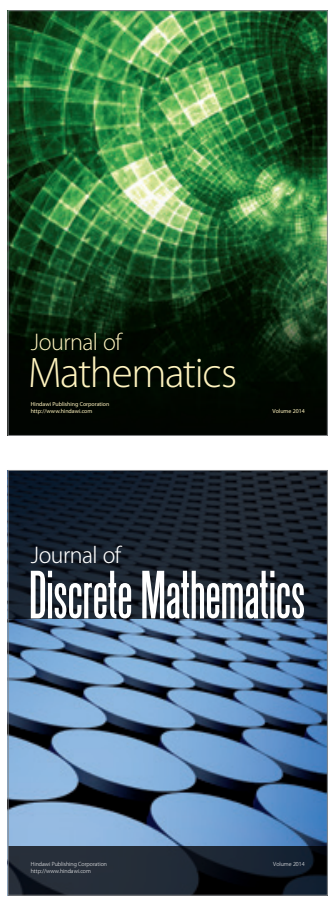

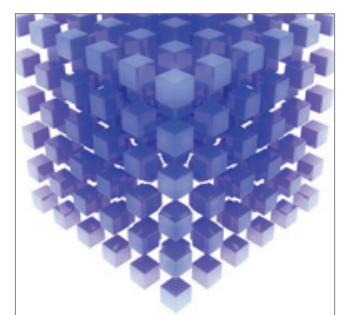

Mathematical Problems in Engineering
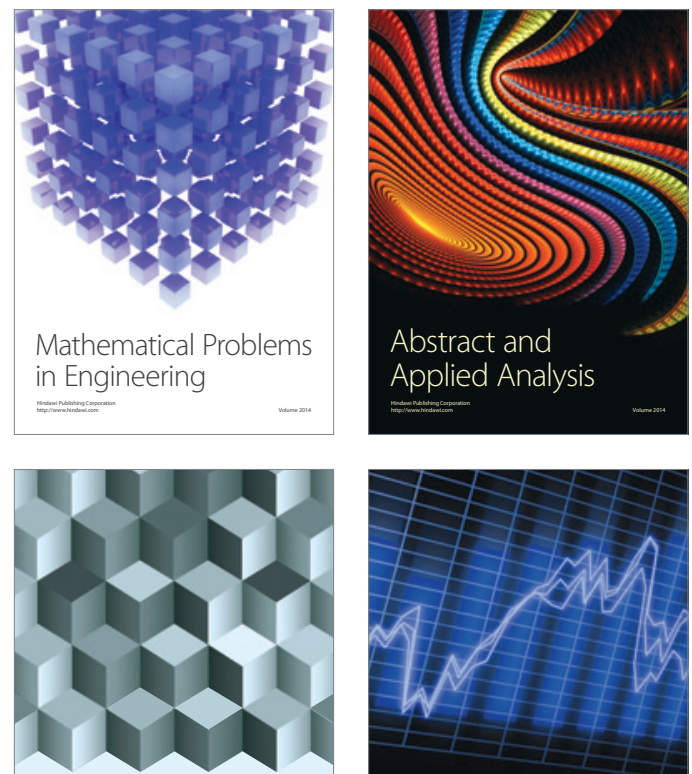

Journal of

Function Spaces

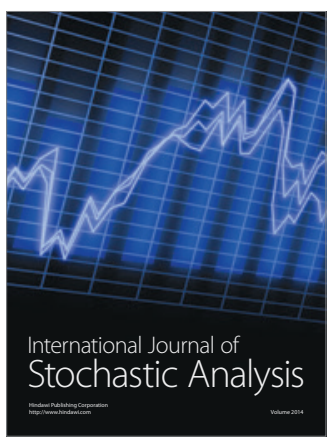

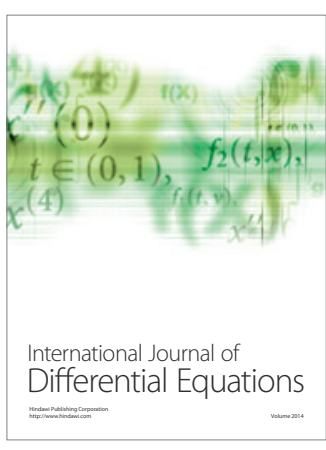
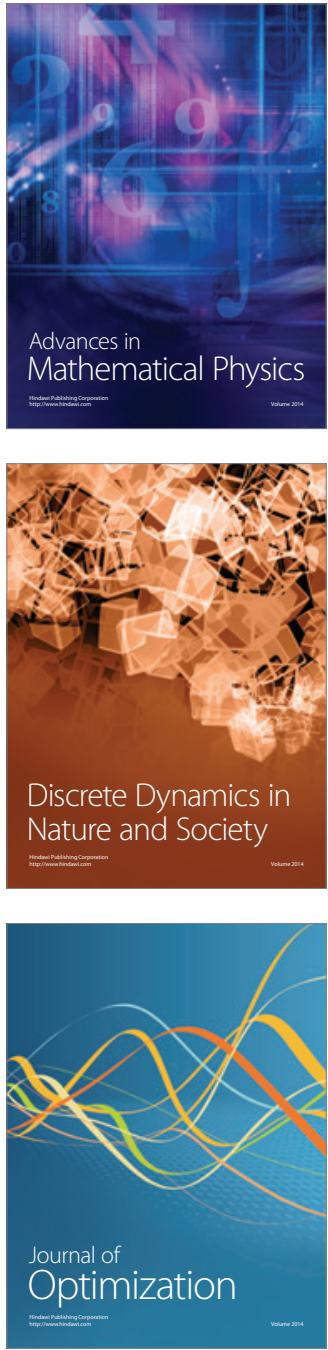